Check for updates

Cite this: RSC Adv., 2019, 9, 15997

Received 15th March 2019

Accepted 13th May 2019

DOI: 10.1039/c9ra02012h

rsc.li/rsc-advances

\section{White light emission from a mixture of silicon quantum dots and gold nanoclusters and its utilities in sensing of mercury(II) ions and thiol containing amino acid $\dagger$}

\begin{abstract}
Swati Tanwar, (D) Bhagwati Sharma, (D) : Vishaldeep Kaur and Tapasi Sen (D) *
White light emitting mixture (WLEM) was produced by controlled mixing of blue emitting silicon quantum dots (Si QDs) and orange red emitting gold nanoclusters (Au NCs). The chromaticity color co-ordinate of the WLEM studied using CIE (Commission Internationale del'Eclairage) diagram was found to be $(0.33$, 0.32), which was very close to that of perfect white light emitting source. The WLEM can also be achieved in the form of gel, solid and film with nearly the same CIE co-ordinates which enhances its utility as white light-emitting source in solid state devices. The reversible and thermo-responsive behaviour of the WLEM broadens its application in thermal sensing. Furthermore, the system was found to be showing fast, sensitive and selective detection of $\mathrm{Hg}^{2+}$ ions and thiol containing amino acid cysteine.
\end{abstract}

\section{Introduction}

The emission of white light from various organic, inorganic and hybrid materials is a highly desired phenomenon of immense interest due to its prospective applications in displays or light emitting devices. ${ }^{1}$ Besides the inorganic electroluminescence devices and fluorescent lamps, white organic light-emitting diodes (OLEDs) have found enormous applications in the development of solid state lighting as alternatives to incandescent lamps. White light-emitting diode (LED) has the potential to reduce consumption of light energy and pollution from fossil fuel power plants by significantly improving lighting efficiency, ${ }^{2}$ and are of great interest due to their long lifetime, small size, and low energy consumption. Presently, commercial white LEDs are mainly achieved by combining rare-earth doped phosphor materials. Commercial WLEDs, developed by mixing yellow phosphor materials with blue LED chips, ${ }^{3,4}$ suffer from some serious drawbacks in terms of their stability such as change in chromaticity and color rendering with time. ${ }^{5}$ Therefore development of novel, energy efficient and reliable white light emitting sources remains a great challenge. Several strategies have been adopted to generate and improve the quality of white light by using different photofunctional sources such as polymers, ${ }^{6}$ metal-organic frameworks, ${ }^{7,8}$ semiconducting quantum dots, ${ }^{9,10}$ lanthanide co-doped systems, ${ }^{11,12}$ organic molecules ${ }^{13,14}$ etc.

Institute of Nano Science and Technology, Mohali, Phase-10, Sector-64, Punjab-160062, India.E-mail: tapasi@inst.ac.in

$\dagger$ Electronic supplementary information (ESI) available. See DOI: 10.1039/c9ra02012h

\$ Present address: Materials Research Centre, Malaviya National Institute of Technology, JLN Marg, Jhalana Gram, Malviya Nagar, Jaipur-302017.
The development of white light emitting mixtures in solution phase is important because of their possible applications due to easy device fabrication on different types of substrates. Several reports exist on various composites such as carbon dots, silica hybrids or boronate microparticles which were exploited as white light emitting materials owing to their easy solution phase synthesis, good stability, bio-compatibility, cost effectiveness and non-toxicity. ${ }^{15-19}$ Mandani et al. demonstrated the solution based synthesis of white light emitting system by controlled mixing of carbon dots with rhodamine $\mathrm{B}$ dye and showed its application in $\mathrm{Fe}^{3+}$ sensing. ${ }^{20}$ Recently, fluorescent silicon quantum dots (Si QDs) have gained considerable interest due to their unique optical, electronic and mechanical properties, and excellent biocompatibility. ${ }^{21-30}$ Fluorescent Si QDs have been used for a myriad of applications, such as in bioimaging, sensing, and optoelectronic devices. ${ }^{28,31,32}$ Although a handful of reports exist in literature where several groups have tried integrating Si QDs into light emitting devices, ${ }^{33-35}$ there are only a few reports showing applicability of Si QDs for generation of white light. In a very recent study, Bose et al. demonstrated the production of white light by mixing green luminescent $\mathrm{Si}$ nanoparticles (NPs) with blue emitting Si NPs and red luminescent Au nanoclusters. ${ }^{36}$ Tu et al. developed WLEDs by mixing red emitting Si QDs with green and blue emitting rare earth elements (REEs) phosphors. ${ }^{37}$ The synthesis of red emitting Si QDs used for development of WLED involved etching of Si wafer with highly corrosive HF solution which is not environment friendly. Ghosh et al. developed a hybrid WLED using red emitting Si nanocrystals and poly[ $N, N^{\prime}$-bis(4-butylphenyl)- $N, N^{\prime}$ bis(phenyl)benzidine] film which played the role of hole transporter as well as blue green light emitter in the device. ${ }^{38}$ 
Luminescent noble metal clusters have received a lot of attention in various fields of nanotechnology due to their high fluorescence, easy synthesis, water solubility, stability, and reduced toxicity. ${ }^{39}$ In particular, gold nanoclusters (Au NCs) have been used as probes for sensing and assays in several studies due to their behaviour like artificial atoms with discrete and size-tuneable electronic transitions from visible to near IR region. ${ }^{40}$ Few reports exist on fluorescent Au NCs used as light emitting sources in optoelectronic devices. For example, Barman et al. reported the composite of carbon dots and dyeencapsulated BSA-protein-capped $\mathrm{Au}$ NCs for white light generation. ${ }^{41}$ Goswami et al. demonstrated the formation of white light emission by combining the blue and green fluorescence of green fluorescent protein (GFP)-expressing bacteria and orange luminescence of Au nanoclusters. ${ }^{42}$ In most of the previous studies, the generation of white light emission was performed by combining different types of dyes or mixing dye with fluorescent NPs or mixing different types of NPs/NCs, which consist of three components. Such systems can suffer from self-absorption, color ageing, non-radiative energy transfer and unwanted changes in the chromaticity coordinates. ${ }^{\mathbf{4 2 , 4 3}}$ So far, to the best of our knowledge, there is no report on the production of white light emission by mixing single emitting NPs and single luminescent NCs.

Herein, we demonstrate a solution phase synthesis of white light emitting mixture (WLEM) by controlled mixing of blue emitting Si QDs and orange red emitting Au NCs at a particular ratio. The WLEM mixture can be incorporated in a gel, thin film as well as solid forms which will have potential utilities in designing solid state devices. Our developed WLEM showed reversible and thermo-responsive behaviour that can broaden its application in the field of thermal sensing. Furthermore, the as obtained WLEM was employed for the reversible and selective detection of analyte molecules such as mercuric $\left(\mathrm{Hg}^{2+}\right)$ ions and cysteine amino acid (Cys). Presently, the ultrasensitive and visual detection of heavy metal ions such as mercuric ions $\left(\mathrm{Hg}^{2+}\right)$ has been a focal point of research because of their tremendous toxicity and potential threats to human health as well as aquatic animals even at nanomolar concentrations due to their high water solubility. ${ }^{\mathbf{4 4 , 4 5}}$ On the other hand, the detection of minute quantities of the amino acid, L-cysteine, in biological fluids is also very important to detect early onset of diseases like edema, liver damage and slow growth rate in children. ${ }^{46}$ It was found that our WLEM can function as a visual sensor for the rapid, sensitive, selective and sequential sensing of $\mathrm{Hg}^{2+}$ ions and cysteine molecule via a reversible color change of the WLEM from white to blue to white again. The fluorescence quenching of $\mathrm{Au}$ NCs component in the WLEM upon addition of $\mathrm{Hg}^{2+}$ ions was due to metallophilic $\mathrm{Hg}^{2+}-\mathrm{Au}^{+}$interactions. ${ }^{47}$ Eventually the white emission of WLEM turned into to blue. The white emission recovered again by subsequent addi-

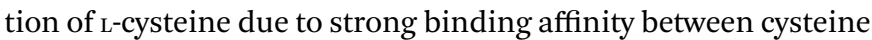
(thiol containing amino acid) and thiophilic metal cation $\mathrm{Hg}^{2+}$ which resulted in weakening of $\mathrm{Hg}^{2+}-\mathrm{Au}^{+}$interactions and subsequent detachment of $\mathrm{Hg}^{2+}$ ions from the surface of $\mathrm{Au}$ NCs. ${ }^{48,49}$ Our developed nontoxic WLEM will have potential applications in selective sensing of a wide range of analyte molecules (chemical or biological species) based on their specific chemical interaction with the individual components (i.e. either QDs or Au NCs) and can also be used in bio-imaging.

\section{Experimental section}

\subsection{Materials}

Gold chloride trihydrate $\left(\mathrm{HAuCl}_{4} \cdot 3 \mathrm{H}_{2} \mathrm{O}\right)$, glutathione (GSH), Lalanine, L-aspartic acid, L-histidine, L-phenylalanine, L-arginine, L-glutamic acid, L-cysteine, polyvinylpyrrolidone (PVP), Dglucose and 3-aminopropyltriethoxysilane (APTES) were purchased from Sigma-Aldrich. Agarose was purchased from Himedia. Carbon coated transmission electron microscopy (TEM) grids used for imaging were purchased from Ted Pella. Dialysis membrane with a molecular weight cut off of $0.5-1 \mathrm{kDa}$ was purchased from Spectrum labs. Dialysis membrane with a molecular weight cut off of 7-10 kDa was purchased from Himedia. Salts used for sensing namely calcium carbonate, ferric nitrate nonahydrate, calcium chloride dihydrate, sodium chloride, zinc nitrate hexahydrate, magnesium chloride hexahydrate, sodium chlorate, cadmium nitrate tetrahydrate, nickel chloride hexahydrate, copper nitrate trihydrate, lead chloride, mercuric chloride, quinine sulphate dihydrate and sulphuric acid were purchased from Merck, India. All the chemicals were of analytical grade and were used without any further purification. Milli-Q water was used throughout the experiments.

\subsection{Instrumentation}

UV-visible absorption spectra were recorded at room temperature using a Shimadzu UV-2600 spectrophotometer. Photoluminescence (PL) spectra were recorded on an FS5 steady state fluorescence spectrometer from Edinburg instruments. Dynamic light scattering (DLS) measurements were performed on a Malvern zetasizer nano ZSP instrument. TEM measurements were performed using a JEOL 2100 microscope with a lanthanum hexaboride $\left(\mathrm{LaB}_{6}\right)$ filament at an accelerating voltage of $200 \mathrm{kV}$. Average nanoparticle size was measured using Gatan microscopy suite software. STEM-EDS mapping images were obtained using a JEOL JEM $2100 \mathrm{~F}$ system at an accelerating voltage of $200 \mathrm{kV}$.

\subsection{Synthesis of blue emitting silicon quantum dots (Si QDs)}

The Si QDs were synthesized according to previously reported protocol by our group. ${ }^{30}$ Briefly, $450 \mathrm{mg}$ of D-glucose was dissolved in $4 \mathrm{~mL}$ of water. To this solution $0.5 \mathrm{~mL}$ of APTES was added slowly under stirring and the stirring was continued at room temperature for 48 hours. Gradually, the color of the solution changed from colorless to dark brown. The solution was dialyzed against Milli-Q water to remove unreacted reactants using a $1 \mathrm{kDa}$ dialysis membrane for 6 hours and stored at room temperature for further use.

\subsection{Synthesis of orange red emitting glutathione (GSH) capped gold nanoclusters (Au NCs)}

The synthesis of GSH capped Au NCs was performed following a reported procedure. ${ }^{\mathbf{5 0}}$ Briefly, freshly prepared aqueous 
solutions of $\mathrm{HAuCl}_{4} \cdot 3 \mathrm{H}_{2} \mathrm{O}(20 \mathrm{mM}, 0.5 \mathrm{~mL})$ and $\mathrm{GSH}(100 \mathrm{mM}$, $0.15 \mathrm{~mL}$ ) were mixed with $4.35 \mathrm{~mL}$ of ultrapure water at $25{ }^{\circ} \mathrm{C}$. The reaction mixture was heated to $70{ }^{\circ} \mathrm{C}$ under gentle stirring (500 rpm) for $24 \mathrm{~h}$ and resulted in a pale yellow colored solution which indicated the formation of Au NCs. The as obtained solution was dialyzed against water using a $7 \mathrm{kDa}$ dialysis membrane and was stored at $4{ }^{\circ} \mathrm{C}$ for further use.

\subsection{Synthesis of white light emitting mixture (WLEM)}

As synthesized solutions of Si QDs and Au NCs were diluted first so that the emission intensity of both the components become almost identical. To generate white light emitting mixture, we have mixed the diluted solutions of Si QDs and Au NCs in different ratio. It was found that white light emission was coming from a mixture of $200 \mu \mathrm{L}$ of Au NCs to $2 \mathrm{~mL}$ solution of Si QDs.

\subsection{Metal ions sensing}

For metal ions sensing, $2 \mathrm{~mL}$ of as obtained WLEM was titrated with $4 \mu \mathrm{L}(1 \mathrm{mM})$ solution of different metal salts. PL spectra of WLEM in presence of different metal ions were recorded after 5 minutes of incubation at room temperature.

\section{Results and discussion}

\subsection{Optical and structural characterizations of Si QDs and Au NCs}

Dynamic light scattering measurement of as synthesized Si QDs indicates the formation of tiny particles with an average hydrodynamic diameter of $3.6 \mathrm{~nm}$ (Fig. S1a $\dagger$ ). The emission spectrum of the Si QDs as presented in Fig. S1b† shows blue emission with a maximum at $464 \mathrm{~nm}$ upon excitation at a wavelength of $365 \mathrm{~nm}$. Structural characterization of Si QDs was further done using TEM study which confirms the formation of Si QDs with an average diameter of $\sim 2.7 \pm 0.3 \mathrm{~nm}$ (Fig. S1c $\dagger$ ), which matches well with the DLS data.

The UV-visible absorption spectrum of the as prepared Au NCs shows a broad absorption in the region of 200 to $400 \mathrm{~nm}$ without appearance of any prominent peak (Fig. S2a $\dagger$ ). The absence of localized surface plasmon resonance band (SPR) in the absorption spectrum reveals the formation of very small sized $\mathrm{Au}$ nanoparticles. The as synthesized $\mathrm{Au}$ NCs showed orange red emission with a maximum centred at $620 \mathrm{~nm}$ upon excitation with $365 \mathrm{~nm}$ wavelength (Fig. S2b $\dagger$ ), indicating the formation of Au nanoclusters. DLS study indicated the formation of particles with an average size of $2.4 \mathrm{~nm}$ (Fig. S2c $\dagger$ ). The size and morphology of the Au NCs were further ascertained from the TEM studies, which confirmed the formation of spherical Au NCs of average size $\sim 1.9 \pm 0.8 \mathrm{~nm}$ (Fig. S2d $\dagger$ ).

\subsection{Photoluminescence properties of WLEM}

To generate white light emitting mixture we have mixed the diluted solutions of Si QDs and Au NCs in different ratio. The PL spectra coming from such mixture solutions have been recorded and shown in Fig. 1a. We have also checked the Commission Internationale d'Eclairage (CIE) chromaticity co-ordinates of pure Si QDs, Au NCs, and their mixtures and compared with that of the perfect white light emitting source. It is well reported that a perfect white light emitting source has CIE chromaticity coordinate of $(0.33,0.33)$. The CIE co-ordinate of as synthesized Si QDs was found to be $(0.21,0.26)$ in the blue region as represented in Fig. 1b. On the other hand, the CIE co-ordinate for

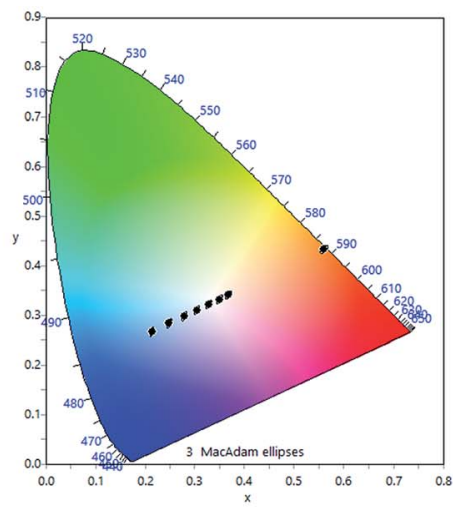

\section{b)}

c)
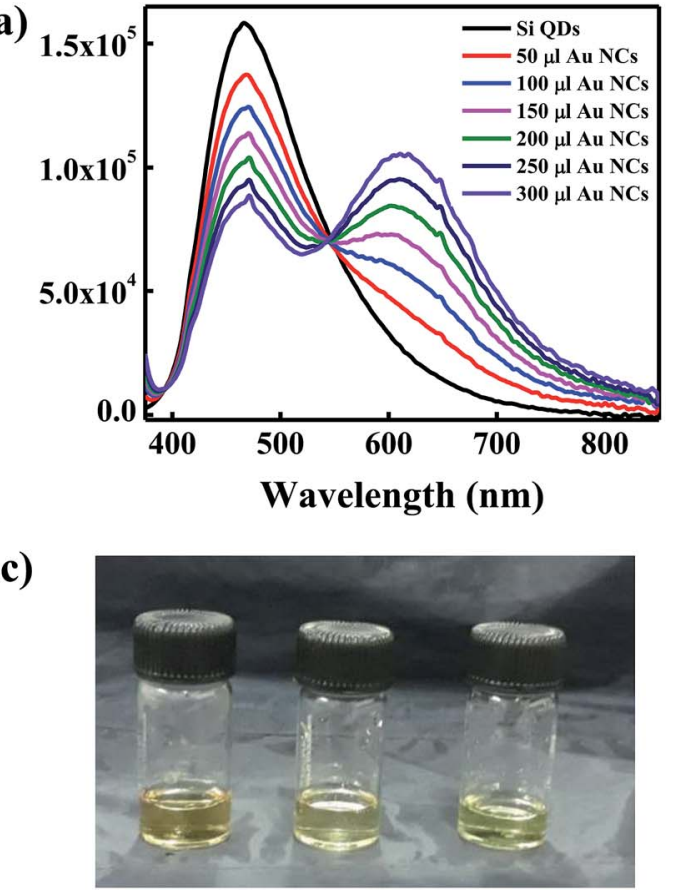

Fig. 1 (a) PL spectra of Si QDs after addition of different volumes of Au NCs; (b) corresponding chromaticity plot for color co-ordinates; (c) digital images of Si QDs, WLEM and Au NCs in day light; and (d) digital images of Si QDs, WLEM and Au NCs under UV light ( $\lambda_{\text {ex }}=365 \mathrm{~nm}$ ). 
the Au NCs was found to be in the red region with value of $(0.56$, 0.43) (Fig. 1b). A careful investigation of the CIE co-ordinates of both Si QDs and Au NCs suggested that the mixing of both components in a particular ratio could cover the entire visible region, thus leading to the generation of white light. The PL spectra of Si QDs upon addition of different volumes of Au NCs have been shown in Fig. 1a. It is clearly visible from the emission spectra that the pattern of the spectrum of Si QDs was changing gradually after addition of Au NCs to the Si QDs solution. The appearance of a new band at $620 \mathrm{~nm}$ was observed upon addition of Au NCs in addition to the initial peak present at $464 \mathrm{~nm}$. It was found that with increasing the concentration of Au NCs the intensity of the Si QDs peak centred at $464 \mathrm{~nm}$ was gradually decreasing along with an increase in the emission intensity of the Au NCs band at $620 \mathrm{~nm}$. A clear iso-emissive point could be observed at $543 \mathrm{~nm}$ indicating the presence of only two emissive species in the system. It was observed that upon addition of Au NCs component, the CIE co-ordinates of the mixture system started moving from blue region toward that of perfect white source i.e. $(0.33,0.33)$ as shown in Fig. $1 \mathrm{~b}$ and Table 1. The analysis of CIE co-ordinates (Fig. 1b) revealed the formation of WLEM with co-ordinate of $(0.33,0.32)$ when $200 \mu \mathrm{L}$ of Au NCs solution was added to $2 \mathrm{~mL}$ solution of Si QDs. This value is pretty close to that of perfect white light emitting source $(0.33,0.33)$. The digital images under daylight and under UV light for blue emitting Si QDs, orange emitting Au NCs and white light emitting mixture are shown in Fig. $1 \mathrm{c}$ and $\mathrm{d}$.

As we have seen from Fig. 1a, addition of increasing volume of $\mathrm{Au}$ NCs to the Si QDs solution led to a quenching in the

Table 1 CIE co-ordinates of Si QDs with different volumes of Au NCs

\begin{tabular}{lll}
\hline Systems & $X$ & $Y$ \\
\hline Si QDs & 0.21 & 0.26 \\
Si QDs $+50 \mu \mathrm{L} \mathrm{Au} \mathrm{NCs}$ & 0.25 & 0.28 \\
Si QDs $+100 \mu \mathrm{L} \mathrm{Au} \mathrm{NCs}$ & 0.29 & 0.30 \\
Si QDs $+150 \mu \mathrm{L} \mathrm{Au} \mathrm{NCs}$ & 0.30 & 0.31 \\
Si QDs $+200 \mu \mathrm{L} \mathrm{Au} \mathrm{NCs}$ & 0.33 & 0.32 \\
Si QDs $+250 \mu \mathrm{L} \mathrm{Au} \mathrm{NCs}$ & 0.35 & 0.33 \\
Si QDs $+300 \mu \mathrm{L} \mathrm{Au} \mathrm{NCs}$ & 0.37 & 0.34 \\
Au NCs & 0.56 & 0.43
\end{tabular}

a)

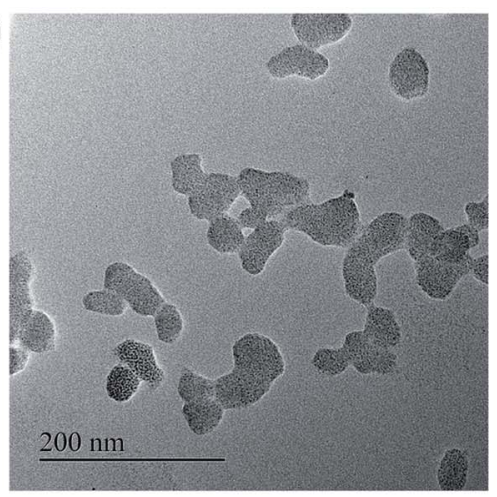

b)

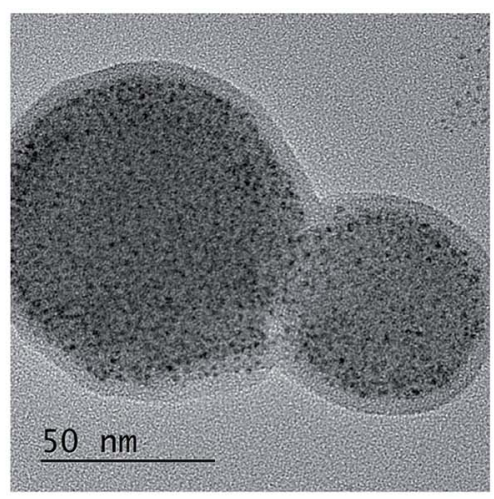

emission of Si QDs at $464 \mathrm{~nm}$. In order to get insights into the nature of quenching, lifetime measurements of Si QDs and WLEM were performed. The lifetime of Si QDs and WLEM were collected at $464 \mathrm{~nm}$ with an excitation wavelength of $402 \mathrm{~nm}$ (Fig. S3†). It was found that lifetime of Si QDs remained almost unaltered in Si QDs solution (9.5 ns) and WLEM (9.8 ns) which further confirmed the involvement of static quenching mechanism behind the PL quenching of Si QDs (Table S1†).

\subsection{Structural characterizations of WLEM}

The as synthesized WLEM was characterized using TEM to get information about the morphology of the mixture. Fig. 2a and $b$ show the low and high resolution TEM images of WLEM which indicate the formation of agglomerated structures of different shapes. The HRTEM images of the agglomerated structures (Fig. 2c) show lattice fringes of $d$-spacing of $0.23 \mathrm{~nm}$ corresponding to (111) plane of $\mathrm{Au}$ and $0.16 \mathrm{~nm}$ corresponding to (311) plane of Si.

The structure formed is similar to that of a core-shell type morphology where the aggregated Au NCs form the core and the Si QDs form a continuous thin shell of thickness around $5 \mathrm{~nm}$. To confirm the nature of the core and the shell, we performed a scanning transmission electron microscopy-energy dispersive X-ray spectroscopy (STEM-EDS) elemental mapping for the WLEM (Fig. S4†). STEM-EDS elemental mapping images confirmed that the Si QDs are homogeneously distributed over $\mathrm{Au}$ NCs in the agglomerated structures and the Au NCs aggregates form the core. The binding of the Si QDs with Au NCs can be attributed to the presence of various functional moieties in GSH that is present on the surface of Au NCs. The formation of aggregates further supports the strong interaction between $\mathrm{Si}$ QDs and $\mathrm{Au}$ NCs which can result in static quenching of PL intensity of Si QDs.

\subsection{Stability studies of WLEM}

For a fluorescent material to be used for applications such as in sensing, it is important for the material to be stable towards light and temperature. Therefore, it was imperative for us to study the stability of the WLEM under these conditions. It was observed that the as synthesized WLEM showed photostability

Fig. 2 (a) and (b) TEM; and (c) HRTEM images of WLEM.

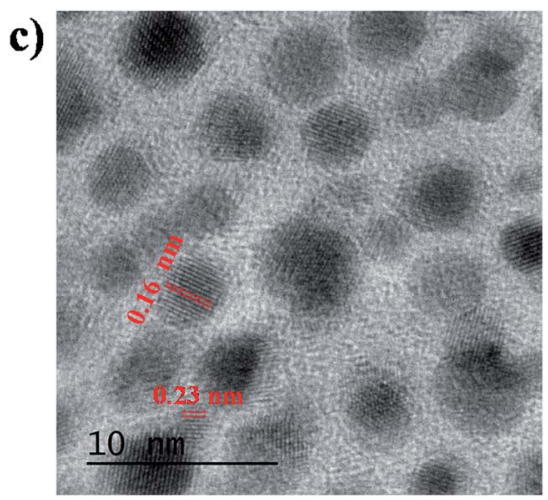


over a longer period of time (more than 3 months) at room temperature, hence, making it a suitable candidate as phosphor material in LED applications.

Optical response of the WLEM toward temperature was studied by recording its temperature dependent PL spectra in the range of $20^{\circ} \mathrm{C}$ to $80^{\circ} \mathrm{C}$ (Fig. S5a ${ }^{\dagger}$ ). It was observed that the PL intensity of the WLEM decreased significantly with increasing temperature from $20{ }^{\circ} \mathrm{C}$ to $80{ }^{\circ} \mathrm{C}$. The emission intensity of Si QDs component present in the WLEM decreased by approximately half of its intensity at $80{ }^{\circ} \mathrm{C}$, whereas the temperature induced PL quenching was more than $90 \%$ in case of $\mathrm{Au}$ NCs component in the WLEM. The higher rate of temperature induced PL quenching for Au NCs compared to Si QDs resulted in shifting of CIE co-ordinates of WLEM towards bluish region (as shown in Fig. S5b†).

Interestingly, it was found that upon cooling the WLEM from $80{ }^{\circ} \mathrm{C}$ to $20^{\circ} \mathrm{C}$, its white light emission properties regained (as shown in Fig. 3a). The reversible thermo responsive behavior can be ascribed to synergistic effects between oxygen rich functional groups present on the surface of QDs and hydrogen bonding with water. ${ }^{30}$ This reversible thermo-response of WLEM was repeated several times and was found to be stable for several heating and cooling cycles (Fig. 3b and c). The reversible thermo-response and water soluble luminescent properties of as synthesized WLEM make it suitable for optical thermometry and thermography applications..$^{51,52}$

\subsection{Optical properties of WLEM}

For practical application of white light emitting materials, it is essential that the white light be generated in solid or in gel state. Therefore, to justify the candidature of WLEM as a potential phosphor material for utilities in WLEDs, white light emission was studied in both gel form and solid state condition. In order to check the emission in gel form, both the components of WLEM were mixed together with melted agarose in the same concentration as those were used for preparing WLEM. After gelification, it was found that gel is also giving white light emission upon UV light illumination (as shown in Fig. 4a). PL spectrum of gel was similar to that of liquid WLEM with CIE co-ordinate of $(0.32,0.30)$ (Fig. $4 \mathrm{~b}$ and c). The solid state emission coming from the WLEM in powder form also showed high quality white light emission with very good CIE co-ordinate $(0.32,0.28)$ (Fig. $4 \mathrm{~d}-\mathrm{f})$. Further, we also studied white light emission from thin film. A thin film was generated by mixing aqueous solutions of PVP, Au NCs and Si QDs in appropriate ratio, which showed white light emission upon illumination by a UV lamp $\left(\lambda_{\text {ex }}=365 \mathrm{~nm}\right.$ ) (Fig. S6 $\dagger$ ). Thus, the WLEM, apart from liquid and solid forms, can also be changed to gel and film states without losing its white light emission property.

\subsection{Application of WLEM for $\mathrm{Hg}^{2+}$ ions and reversible thiol sensing}

White light emitting mixtures are of great interest for sensing applications as they can easily undergo a change in color upon interaction with a particular analyte of interest because of the presence of two or three components in WLEMs. Heavy metal ions like $\mathrm{Hg}^{2+}$ ions are highly toxic to both human and aquatic animals and is known as notorious environmental pollutant. Therefore, we were interested in sensing $\mathrm{Hg}^{2+}$ ions using our WLEM. It is well established that the interaction of $\mathrm{Hg}^{2+}$ ions with Au NCs leads to quenching of emission of Au NCs due to $\mathrm{d}^{10}-\mathrm{d}^{10}$ interaction. ${ }^{47}$ Therefore, it was reasonable to speculate that the interaction of $\mathrm{Hg}^{2+}$ ions with WLEM would lead to quenching of emission of Au NCs, in turn changing the color of emission of the mixture. When different concentrations of $\mathrm{Hg}^{2+}$ ions were added to the WLEM, a gradual decrease in peak centred at $620 \mathrm{~nm}$ due to Au NCs (Fig. 5a) was obtained with increasing concentration of $\mathrm{Hg}^{2+}$ ions. On the other hand, the emission due to Si QDs at $464 \mathrm{~nm}$ remained unaltered. Upon illumination of the mixture under UV lamp, it was observed that the color of the mixture changed from white to blue after addition of $640 \mathrm{nM} \mathrm{Hg}^{2+}$ ions. The emission of Au NCs was quenched due to its specific interaction with $\mathrm{Hg}^{2+}$ ions, which resulted in the emission of Si QDs only to observe. The present method is fast and sensitive toward sensing of $\mathrm{Hg}^{2+}$ ions and the limit of detection was found to be $10 \mathrm{nM}$ (Fig. 5a). Comparison of previously developed small molecular sensors for detection of $\mathrm{Hg}^{2+}$ ions shown in Table 2 indicates that our system is showing better sensitivity than the previously reported ones. Further, the selectivity of the present system was also studied by recording the PL spectra of the WLEM in the presence of different metal cations and anions with a concentration of $1 \mathrm{mM}$. It was observed that the addition of metal anions and
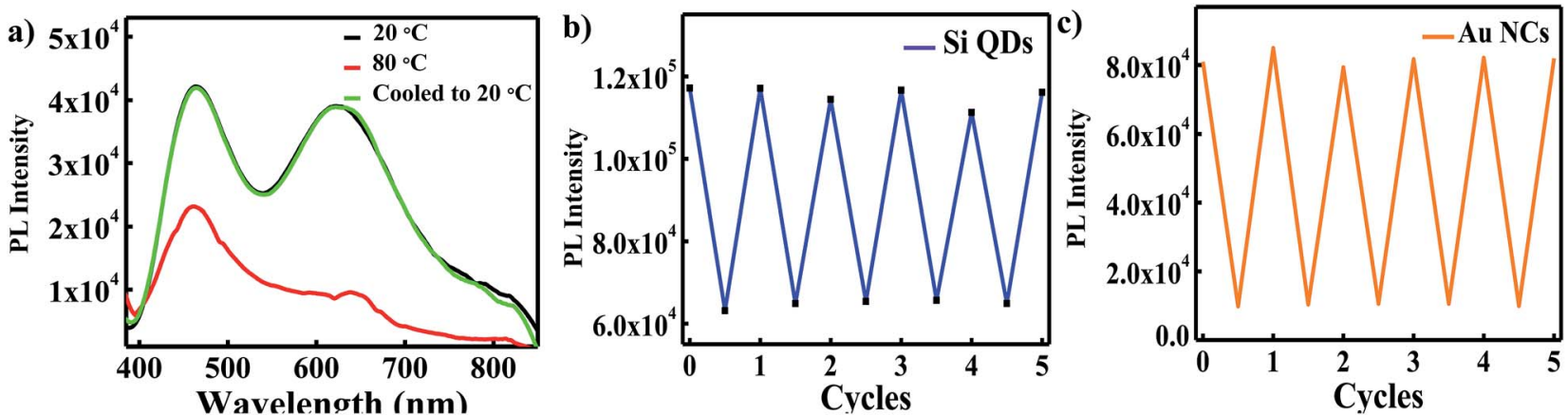

Fig. 3 (a) PL spectra of WLEM at $20^{\circ} \mathrm{C}$ and $80^{\circ} \mathrm{C}$; (b) and (c) change in PL intensity of Si QDs and Au NCs in WLEM during consecutive heatingcooling cycles. 
a)

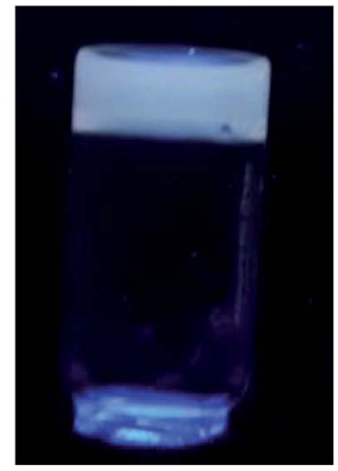

d)

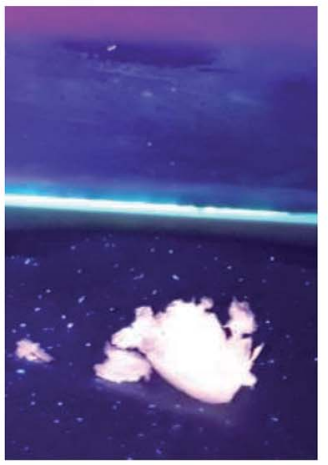

b)

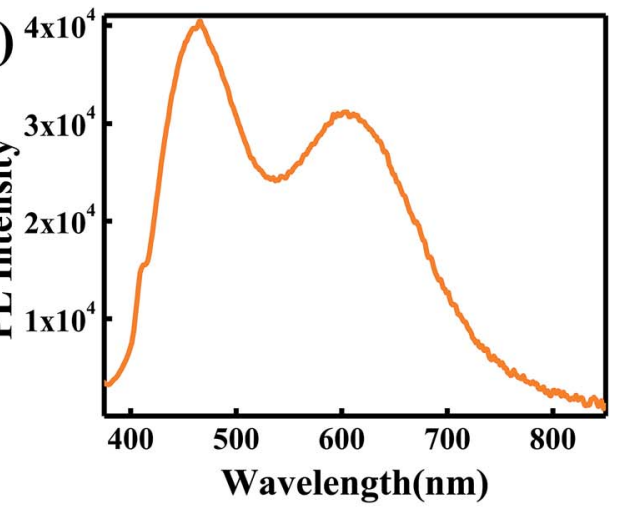

e)

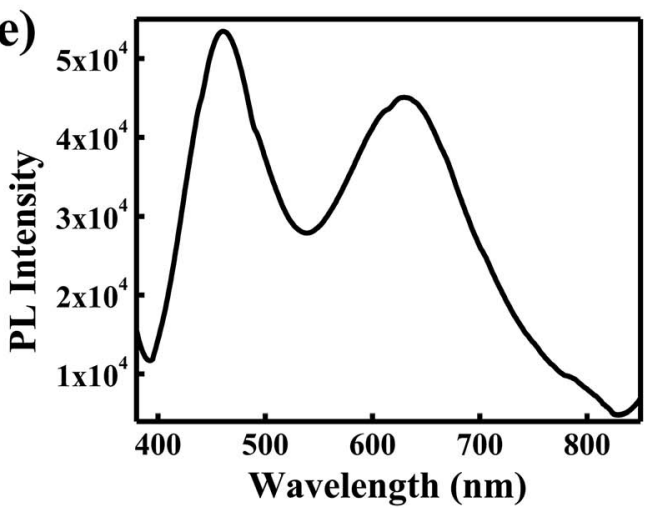

c)

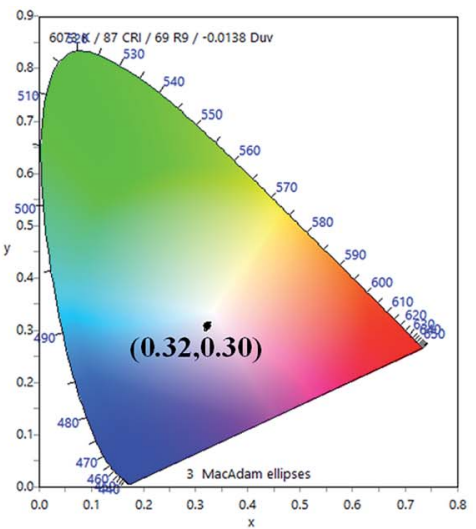

f)

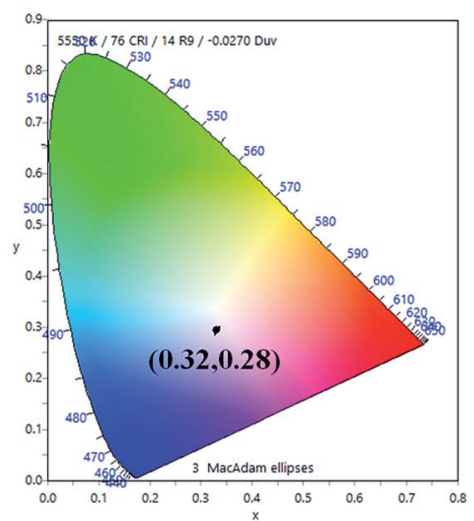

Fig. 4 (a) Digital image of WLEM incorporated in agarose gel under UV light ( $\lambda_{\text {ex }}=365 \mathrm{~nm}$ ); (b) PL spectrum of WLEM incorporated in agarose gel; (c) corresponding chromaticity plot for color co-ordinate; (d) digital image of WLEM incorporated in powder form under UV light $(\lambda$ ex $=365$ $\mathrm{nm}$ ); (e) PL spectrum of WLEM incorporated in powder form; and (f) corresponding chromaticity plot for color co-ordinate.

cations such as $\mathrm{CO}_{3}{ }^{2-}, \mathrm{Fe}^{3+}, \mathrm{Ca}^{2+}, \mathrm{Na}^{+}, \mathrm{Zn}^{2+}, \mathrm{Mg}^{2+}, \mathrm{ClO}_{3}{ }^{-}, \mathrm{Cd}^{2+}$, $\mathrm{Ni}^{2+}, \mathrm{Cu}^{2+}$, and $\mathrm{Pb}^{2+}$ showed no significant change in the PL intensity of WLEM (Fig. 5b), indicating high selectivity of the system for $\mathrm{Hg}^{2+}$ ions. Thus the present system could be employed for the fast, selective, sensitive and visual sensing of $\mathrm{Hg}^{2+}$ ions. Interestingly, the white light emission can fully be recovered again by the subsequent addition of a thiol containing amino acid Cys with a limit of detection of $10 \mathrm{nM}$ (Fig. 5c). Comparative analysis of other known molecular sensors for Cys detection (Table 3) indicates higher sensitivity of our system than the previously reported ones. Digital images of WLEM, WLEM with $\mathrm{Hg}^{2+}$ and WLEM with $\mathrm{Hg}^{2+}$ ions and Cys under UV light are shown in Fig. 6. Thiols have a very strong binding affinity toward $\mathrm{Hg}^{2+}$ ions. Thus, the rapid recovery of white light emission can be attributed to the binding of Cys with $\mathrm{Hg}^{2+}$ ions. Since, $\mathrm{Hg}^{2+}$ ions are now bound to the thiol group in Cys, the $\mathrm{d}^{10}-\mathrm{d}^{10}$ interaction of $\mathrm{Hg}^{2+}$ ions with the $\mathrm{Au}^{+}$ion on the surface of the Au NCs is obviously weakened, thus leading to regain the emission of Au NCs, which in turn leads to recovery of the white emission of the mixture. ${ }^{48}$ This reversible "ON-OFF" of white light emission was found to be very specific to Cys residue only. Scheme 1 illustrates the mechanism of reversible "ON-OFF" PL quenching of Au NCs in WLEM in presence of $\mathrm{Hg}^{2+}$ ions and Cys. The control experiments were performed by taking
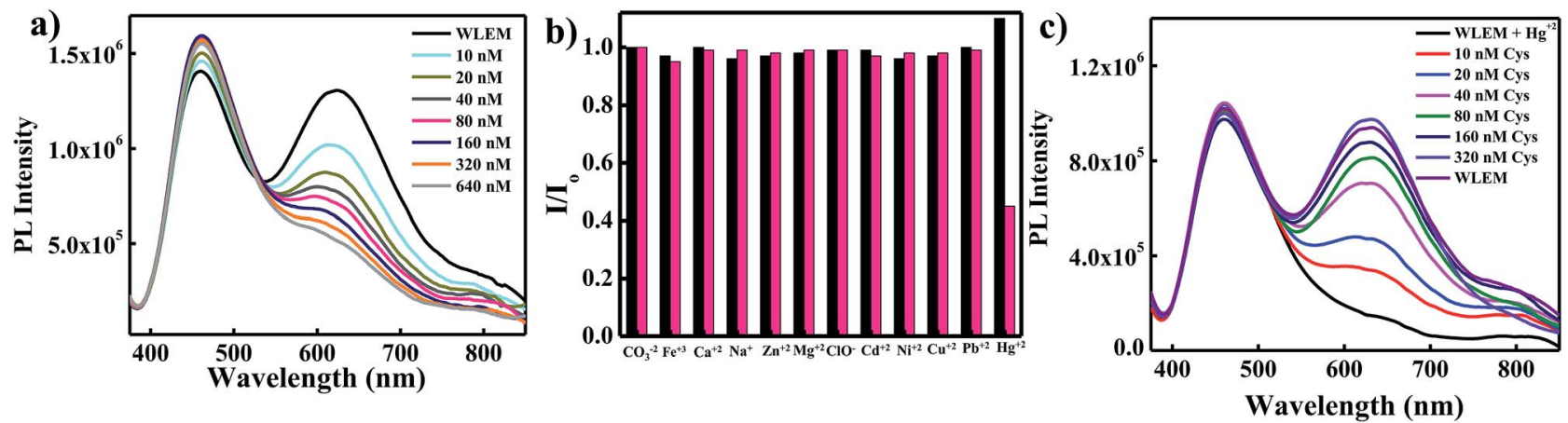

Fig. 5 (a) PL spectra of WLEM upon addition of different concentration of $\mathrm{Hg}^{2+}$ ions; and (b) change in PL intensity of Si QDs (black) and Au NCs (pink) in the WLEM upon addition of different ions; and (c) PL spectra of WLEM having $\mathrm{Hg}^{2+}$ ions with different concentration of Cys. 
Table 2 Comparison of molecular sensors for mercury detection ${ }^{a}$

\begin{tabular}{|c|c|c|c|}
\hline Material & Chemical probe & LOD & Reference \\
\hline \multirow[t]{2}{*}{ Quantum dots } & CdS quantum dots & $25.2 \mathrm{ng} \mathrm{mL}^{-1}$ & Anal. Methods, 2016, 8, 6512-6519 \\
\hline & Nitrogen doped carbon dots & $0.65 \mu \mathrm{M}$ & New J. Chem., 2018, 42, 6824-6830 \\
\hline & Azobenzene & $20 \mu \mathrm{M}$ & Chem.-Eur. J., 2011, 17, 7276-7281 \\
\hline & Phenoxazinone & $100 \mathrm{nM}$ & J. Am. Chem. Soc., 2003, 125, 3418-3419 \\
\hline Biomolecules & DNA oligonucleotides & $40 \mathrm{nM}$ & Angew. Chem., Int. Ed., 2004, 43, 4300-4302 \\
\hline Nanoparticles & Gold nanoparticles & $100 \mathrm{nM}$ & Angew. Chem., Int. Ed., 2007, 46, 4093-4096 \\
\hline \multirow[t]{2}{*}{ Polymers } & Polythiophene with thymine moiety & $30 \mu \mathrm{M}$ & Macromol. Rapid Commun., 2006, 27, 389-392 \\
\hline & $\begin{array}{l}\text { Poly }\left(3-\left(3^{\prime}-N, N, N \text {-triethylamino- } 1^{\prime} \text {-propyloxy)- }\right.\right. \\
\text { 4-methyl-2,5-thiophene hydrochloride) }\end{array}$ & $42 \mathrm{nM}$ & Adv. Mater., 2007, 19, 1471-1474 \\
\hline
\end{tabular}

Table 3 Comparison of molecular sensors for Cys detection

\begin{tabular}{|c|c|c|c|}
\hline Material & Chemical probe & LOD & Reference \\
\hline \multirow[t]{3}{*}{ Organic molecules } & Cinnamaldehyde and pyrimidine & $0.10 \mu \mathrm{M}$ & Sens. Actuators, B, 2018, 255, 2756-2763 \\
\hline & Curcumin & $1 \mu \mathrm{M}$ & Anal. Methods, 2013,5, 3965-3969 \\
\hline & Spiropyran & $40 \mathrm{nM}$ & Angew. Chem., Int. Ed., 2006, 45, 4944-4948 \\
\hline \multirow[t]{3}{*}{ Nanoparticles } & Gold nanorods & Micromolar range & J. Am. Chem. Soc., 2005, 127, 6516-6517 \\
\hline & Nickel oxide nanoflowers & $1.1 \mu \mathrm{M}$ & J. Mater. Chem. B, 2014, 2, 6097 \\
\hline & Gold nanoparticles & $10-100 \mu \mathrm{M}$ & Anal. Chim. Acta, 2010, 671, 80-84 \\
\hline \multirow[t]{2}{*}{ Polymer } & Polythiophene & $12.6 \mu \mathrm{M}$ & Sens. Actuators, B, 2016, 232, 448-453 \\
\hline & $\begin{array}{l}\text { Poly[3-(3- } N, N \text {-diacetateaminopropoxy)-4-methyl } \\
\text { thiophene disodium salts] }\end{array}$ & $0.33 \mu \mathrm{M}$ & Talanta, 2019, 198, 128-136 \\
\hline \multirow[t]{2}{*}{ Quantum dots } & Methyl viologen coated CdS QDs & $0.1 \mu \mathrm{M}$ & Small, 2011, 7, 1624-1628 \\
\hline & CdTe QDs & $0.87 \mu \mathrm{M}$ & Talanta, 2009, 77, 1654-1659 \\
\hline Biomolecules & DNA-gold nanoparticles conjugate & $100 \mathrm{nM}$ & Nano Lett., 2008, 8, 2, 529-533 \\
\hline
\end{tabular}
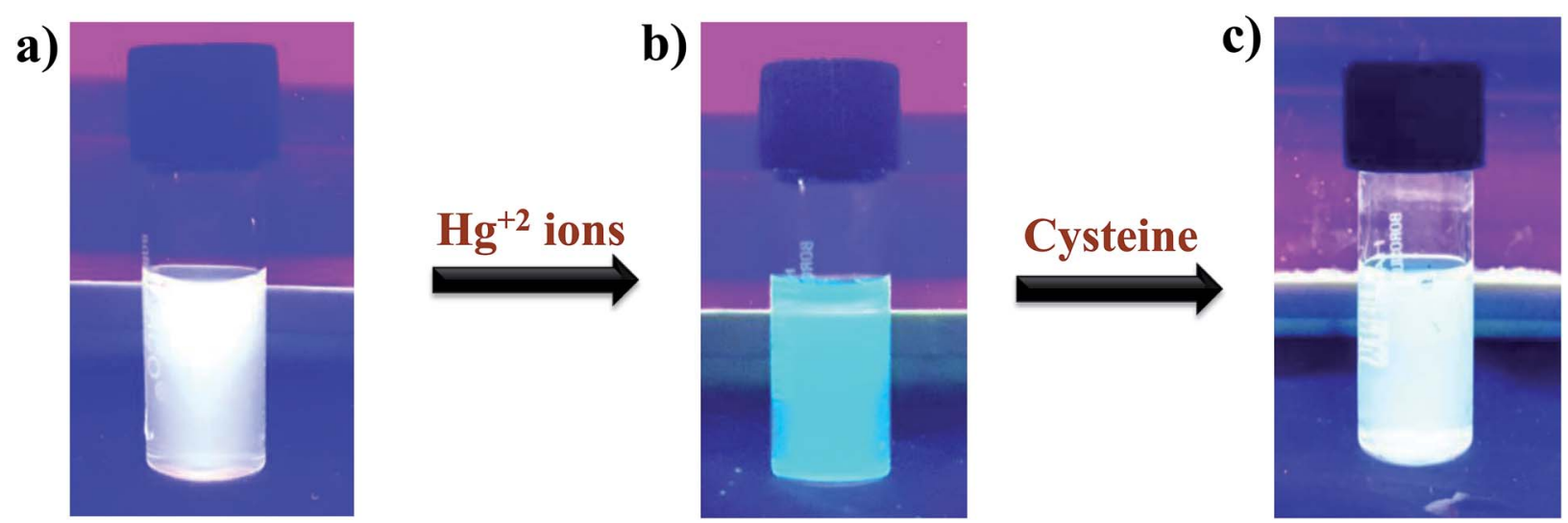

Fig. 6 Digital images of (a) WLEM; (b) WLEM with $\mathrm{Hg}^{2+}$; and (c) WLEM with $\mathrm{Hg}^{2+}$ ions and Cys under UV light $\left(\lambda_{\mathrm{ex}}=365 \mathrm{~nm}\right.$ ).

different non-thiol amino acids such as alanine, aspartic acid, histidine, phenylalanine, arginine, and glutamic acid; however, no change in PL intensity was observed as shown in Fig. 7. To get better insights into ion-induced structural changes, TEM imaging of WLEMs after addition of $\mathrm{Hg}^{2+}$ ions followed by Cys addition was done. TEM images represented in Fig. $\mathrm{S} 7 \dagger$ revealed the distortion of agglomerated structures of WLEM upon addition of $\mathrm{Hg}^{2+}$ ions, this further validates our hypothesis of dissolution of Au NCs structures due to binding of $\mathrm{Hg}^{2+}$ ions with GSH (Fig. S7a and $\mathrm{b} \dagger$ ). Furthermore, upon introduction of Cys to this WLEM with $\mathrm{Hg}^{2+}$ ions, reformation of agglomerated structures take place exactly like pure WLEM (TEM images shown in Fig. S7c and $d \dagger$ ), which results in recovery of white light emission from the mixture. 


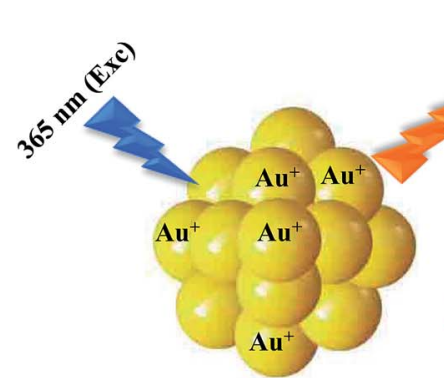

Au NCs
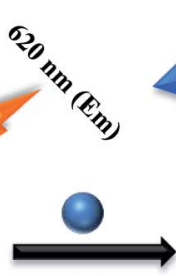

$\mathrm{Hg}^{+2}$ ions

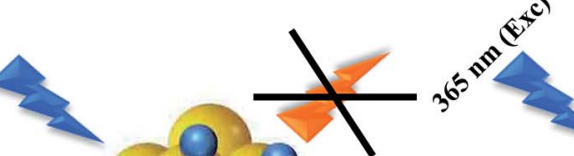

$\infty 00$ ( (1)

\section{Au NCs with $\mathrm{Hg}^{+2}$ ions}

$\sigma_{0}$<smiles>[CH][C@@H]1[CH]C1</smiles>

Cysteine
$+O-\mathrm{Cys}$

$\mathrm{Au}^{+} \mathrm{Au}^{+}$

$\mathrm{Au}^{+} \quad \mathrm{Au}^{+}$

$\mathrm{Hg}^{+2}$ ion-Cys

Au NCs complex

Scheme 1 Schematic depiction of reversible "ON-OFF" PL quenching of Au NCs in WLEM.
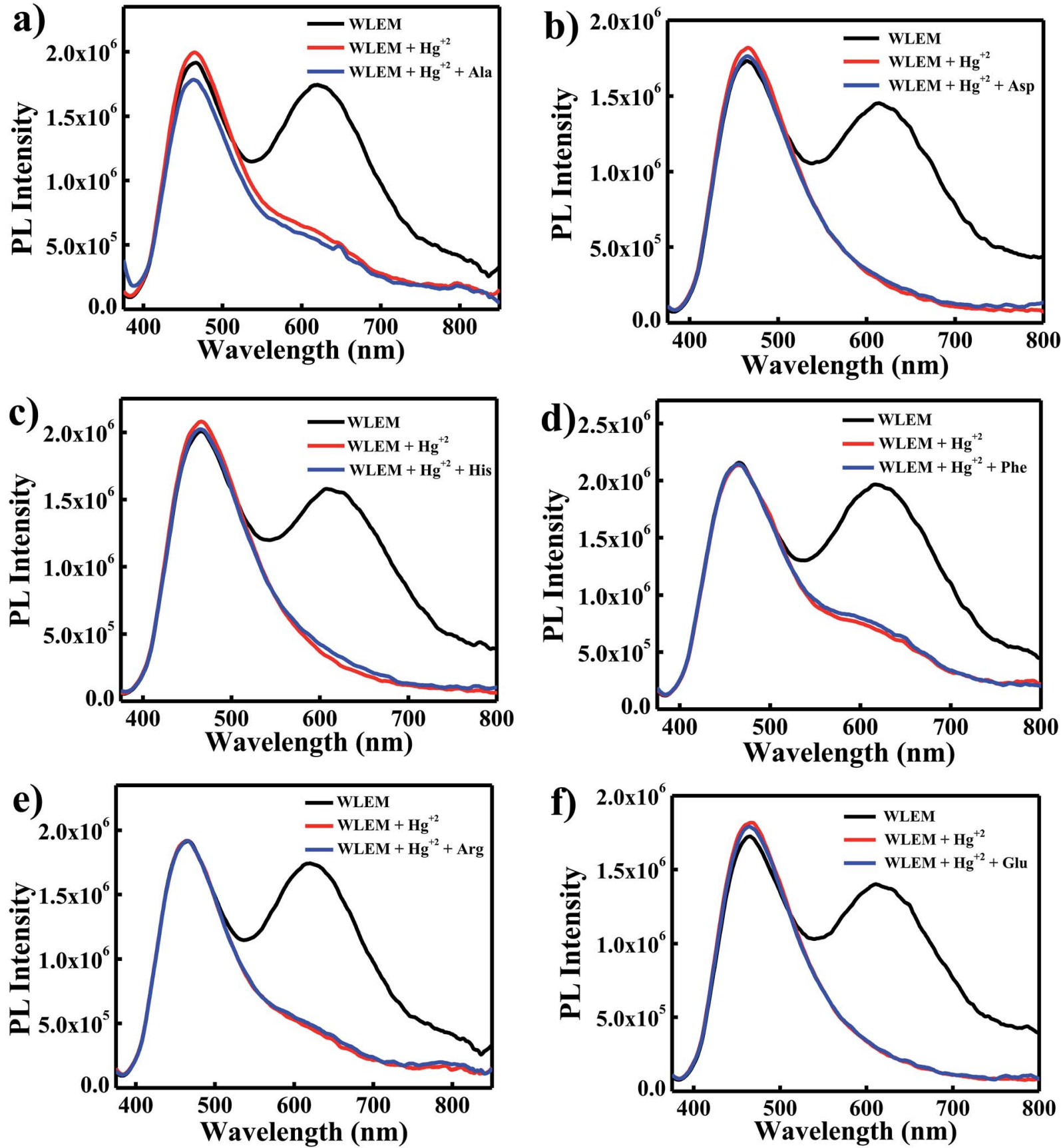

Fig. 7 Fluorescence intensity change of WLEM with $\mathrm{Hg}^{2+}$ ions in presence of different amino acids (a) alanine; (b) aspartic acid; (c) histidine; (d) phenylalanine; (e) arginine; and (f) glutamic acid. 


\section{Conclusions}

In summary, we have designed a simple solution based approach for generation of WLEM. As generated WLEM can be converted to solid, gel or in the form of film, making it a suitable candidate for solid state devices. Furthermore, this system was found to be showing fast, sensitive and selective sensing of $\mathrm{Hg}^{2+}$ ions and thiol containing amino acid Cys. The reversible thermo-response property of WLEM could be utilized in optical thermometry and thermography applications. Such biofriendly white light emitting material holds promise for being used in selective sensing of a wide range of chemical or biological species based on their specific chemical interaction with the individual components present in the WLEM and also in bioimaging.

\section{Conflicts of interest}

There are no conflicts to declare.

\section{Acknowledgements}

T. S. is thankful to Department of Science and Technology (DST) (SERB Project-ECR/2016/000436) for financial support. B. S. and S. T. acknowledge INST for Postdoctoral and Senior Research Fellowships, respectively. V. K. is thankful to DST-SERB project for providing Junior Research Fellowship. T. S. acknowledges Advanced Instrumentation Research Facility (AIRF), Jawaharlal Nehru University (JNU), New Delhi for providing access to the TEM facility and Gajender Saini for technical help.

\section{References}

1 J. Karpiuk, E. Karolak and J. Nowacki, Phys. Chem. Chem. Phys., 2010, 12, 8804-8809.

2 N. Guo, Y. Huang, M. Yang, Y. Song, Y. Zheng and H. You, Phys. Chem. Chem. Phys., 2011, 13, 15077-15082.

3 S. Pimputkar, J. S. Speck, S. P. DenBaars and S. Nakamura, Nat. Photonics, 2009, 3, 180.

4 E. F. Schubert and J. K. Kim, Science, 2005, 308, 1274-1278.

5 N. C. George, K. A. Denault and R. Seshadri, Annu. Rev. Mater. Res., 2013, 43, 481-501.

6 H. Wu, L. Ying, W. Yang and Y. Cao, Chem. Soc. Rev., 2009, 38, 3391-3400.

7 W. Xie, S.-R. Zhang, D.-Y. Du, J.-S. Qin, S.-J. Bao, J. Li, Z.-M. Su, W.-W. He, Q. Fu and Y.-Q. Lan, Inorg. Chem., 2015, 54, 3290-3296.

8 Q.-Y. Yang, K. Wu, J.-J. Jiang, C.-W. Hsu, M. Pan, J.-M. Lehn and C.-Y. Su, Chem. Commun., 2014, 50, 7702-7704.

9 S. K. Panda, S. G. Hickey, H. V. Demir and A. Eychmüller, Angew. Chem., Int. Ed., 2011, 50, 4432-4436.

10 M. J. Bowers, J. R. McBride and S. J. Rosenthal, J. Am. Chem. Soc., 2005, 127, 15378-15379.

11 Y. Ledemi, A.-A. Trudel, V. A. G. Rivera, S. Chenu, E. Veron, L. A. Nunes, M. Allix and Y. Messaddeq, J. Mater. Chem. C, 2014, 2, 5046-5056.
12 S. Dang, J.-H. Zhang and Z.-M. Sun, J. Mater. Chem., 2012, 22, 8868-8873.

13 P. Malakar, D. Modak and E. Prasad, Chem. Commun., 2016, 52, 4309-4312.

14 Q.-Y. Yang and J.-M. Lehn, Angew. Chem., Int. Ed., 2014, 53, 4572-4577.

15 H. Li, Z. Kang, Y. Liu and S.-T. Lee, J. Mater. Chem., 2012, 22, 24230-24253.

16 J. Joseph and A. A. Anappara, J. Lumin., 2016, 178, 128-133.

17 J. Joseph and A. A. Anappara, ChemPhysChem, 2017, 18, 292298.

18 R. Nishiyabu, Y. Sugino and Y. Kubo, Chem. Commun., 2013, 49, 9869-9871.

19 F. d. J. Trindade, E. R. Triboni, B. Castanheira and S. Brochsztain, J. Phys. Chem. C, 2015, 119, 26989-26998.

20 S. Mandani, B. Sharma, D. Dey and T. K. Sarma, RSC Adv., 2016, 6, 84599-84603.

21 N. O'Farrell, A. Houlton and B. R. Horrocks, Int. J. Nanomed., 2006, 1, 451-472.

22 L. Pavesi, L. Dal Negro, C. Mazzoleni, G. Franzò and F. Priolo, Nature, 2000, 408, 440.

23 Y. He, S. Su, T. Xu, Y. Zhong, J. A. Zapien, J. Li, C. Fan and S.-T. Lee, Nano Today, 2011, 6, 122-130.

24 Y. Zhong, F. Peng, X. Wei, Y. Zhou, J. Wang, X. Jiang, Y. Su, S. Su, S.-T. Lee and Y. He, Angew. Chem., Int. Ed., 2012, 51, 8485-8489.

25 Z. Kang, Y. Liu and S.-T. Lee, Nanoscale, 2011, 3, 777-791.

26 X. Cheng, S. B. Lowe, P. J. Reece and J. J. Gooding, Chem. Soc. Rev., 2014, 43, 2680-2700.

27 Y. Su, X. Wei, F. Peng, Y. Zhong, Y. Lu, S. Su, T. Xu, S.-T. Lee and Y. He, Nano Lett., 2012, 12, 1845-1850.

28 Y. Zhong, F. Peng, F. Bao, S. Wang, X. Ji, L. Yang, Y. Su, S.-T. Lee and Y. He, J. Am. Chem. Soc., 2013, 135, 8350-8356.

29 J. Wang, D.-X. Ye, G.-H. Liang, J. Chang, J.-L. Kong and J.-Y. Chen, J. Mater. Chem. B, 2014, 2, 4338-4345.

30 B. Sharma, S. Tanwar and T. Sen, ACS Sustainable Chem. Eng., 2019, 7, 3309-3318.

31 Y. Zhong, X. Sun, S. Wang, F. Peng, F. Bao, Y. Su, Y. Li, S.-T. Lee and Y. He, ACS Nano, 2015, 9, 5958-5967.

32 R. Ban, F. Zheng and J. Zhang, Anal. Methods, 2015, 7, 17321737.

33 K.-Y. Cheng, R. Anthony, U. R. Kortshagen and R. J. Holmes, Nano Lett., 2011, 11, 1952-1956.

34 M. L. Mastronardi, E. J. Henderson, D. P. Puzzo, Y. Chang, Z. B. Wang, M. G. Helander, J. Jeong, N. P. Kherani, Z. Lu and G. A. Ozin, Small, 2012, 8, 3647-3654.

35 F. Maier-Flaig, J. Rinck, M. Stephan, T. Bocksrocker, M. Bruns, C. Kübel, A. K. Powell, G. A. Ozin and U. Lemmer, Nano Lett., 2013, 13, 475-480.

36 S. Bose, M. A. Ganayee, B. Mondal, A. Baidya, S. Chennu, J. S. Mohanty and T. Pradeep, ACS Sustainable Chem. Eng., 2018, 6, 6203-6210.

37 C.-C. Tu, J. H. Hoo, K. F. Böhringer, L. Y. Lin and G. Cao, Opt. Express, 2014, 22, A276-A281.

38 B. Ghosh, Y. Masuda, Y. Wakayama, Y. Imanaka, J.-i. Inoue, K. Hashi, K. Deguchi, H. Yamada, Y. Sakka, S. Ohki, 
T. Shimizu and N. Shirahata, Adv. Funct. Mater., 2014, 24, 7151-7160.

39 X. Qu, Y. Li, L. Li, Y. Wang, J. Liang and J. Liang, J. Nanomater., 2015, 2015, 23.

40 J. Zheng, C. Zhang and R. M. Dickson, Phys. Rev. Lett., 2004, 93, 077402.

41 M. K. Barman, B. Paramanik, D. Bain and A. Patra, Chem.Eur. J., 2016, 22, 11699-11705.

42 U. Goswami, S. Basu, A. Paul, S. S. Ghosh and A. Chattopadhyay, J. Mater. Chem. C, 2017, 5, 12360-12364.

43 S. Bhandari, S. Pramanik, R. Khandelia and A. Chattopadhyay, ACS Appl. Mater. Interfaces, 2016, 8, 1600-1605.

44 W. Zheng, M. Aschner and J.-F. Ghersi-Egea, Toxicol. Appl. Pharmacol., 2003, 192, 1-11.
45 E. M. Nolan and S. J. Lippard, Chem. Rev., 2008, 108, 34433480.

46 S. Shahrokhian, Anal. Chem., 2001, 73, 5972-5978.

47 J. Xie, Y. Zheng and J. Y. Ying, Chem. Commun., 2010, 46, 961-963.

48 J. Wu, R. Sheng, W. Liu, P. Wang, J. Ma, H. Zhang and X. Zhuang, Inorg. Chem., 2011, 50, 6543-6551.

49 Y.-B. Ruan, A.-F. Li, J.-S. Zhao, J.-S. Shen and Y.-B. Jiang, Chem. Commun., 2010, 46, 4938-4940.

50 Z. Luo, X. Yuan, Y. Yu, Q. Zhang, D. T. Leong, J. Y. Lee and J. Xie, J. Am. Chem. Soc., 2012, 134, 16662-16670.

51 E. J. McLaurin, L. R. Bradshaw and D. R. Gamelin, Chem. Mater., 2013, 25, 1283-1292.

52 C. D. S. Brites, P. P. Lima, N. J. O. Silva, A. Millan, V. S. Amaral, F. Palacio and L. D. Carlos, Nanoscale, 2012, 4, 4799-4829. 